\title{
Franchissement d'un seuil : interdit et transgression dans Le rivage des Syrtes de Julien Gracq
}

\section{Threshold Crossing: Forbidden and Transgression in Le rivage des Syrtes by Julien Gracq}

\author{
Martina Petrovičová [64456@mail.muni.cz] \\ Masarykova univerzita, République tchèque
}

\section{RÉSUMÉ}

Le processus initiatique du héros du roman Le rivage des Syrtes est caractérisé par la recherche d'une connaissance complète du monde et de son sens caché. Ainsi, le héros connaît une succession d'épreuves, des limites à franchir qui permettent l'accession à une révélation ontologique. Le but de notre propos sera de montrer comment le franchissement d'un seuil et la transgression de l'interdit représentent des actes catalyseurs de l'initiation.

\section{MoTS CLÉS}

frontière spatiale ; frontière sémantique ; seuil ; transgression ; interdit ; révélation, initiation

\begin{abstract}
The initiation process of the main hero of the novel Le rivage des Syrtes is typical by the search of a higher knowledge of the universe and of its sense manifested beyond visible. Hence, the main hero has to deal with a succession of hardships and with limits to be reached and crossed in order to access to an ontological revelation. The aim of the present article is to demonstrate how reaching of the border and passing of a forbidden threshold represent an effect catalyst in the initiation process.
\end{abstract}

\section{KEYWORDS}

space border; semantic border; treshold; transgression; forbidden; revelation; initiation

REÇU 2016-12-15; ACCEPTE 2017-01-12 
Le présent propos se voudrait une application de la conception sémantique de l'espace qui met au point la différence entre l'étendu et le caractère de l'espace artistique et de l'espace d'un monde extérieur à l’ouvre. Même si la qualité intrinsèque de l'espace artistique est la finitude, il reproduit l'espace réel qui, contrairement, est dans sa nature illimité (Lotman 1973). Cette idée porte l'attention au problème de l'espace artistique. Youri Lotman met en relief l'importance de la modélisation spatiale qui est propre aux textes littéraires et qui s'inspire des structures spatiales extralittéraires. Ce sont les relations spatiales nées de ces structures qui seront au centre de notre attention. Nous visons à démontrer, sur les exemples puisés dans le roman Le rivage des Syrtes, comment l'espace romanesque reproduisant le monde matériel se rattache au continuum spatial du texte caractérisé par les frontières sémantiques.

Selon Lotman, il existe la " possibilité d'une modélisation spatiale de concepts, qui n’ont pas en soi une nature spatiale » $(1973: 310)$. Effectivement, le modèle spatial du monde existant hors du cadre littéraire et qui est caractérisé par la dynamique des oppositions telles que : haut - bas, droit - gauche, ouvert - fermé etc., servent souvent pour designer d'une façon métaphorique les qualités sans aucun contenu spatial, telles que des qualités morales ou des notions de validité ou de non-validité. C'est-à-dire que les traits spatiaux participent ainsi à la modélisation des relations et de la syntagmatique des éléments textuels aussi au niveau idéologique. En d'autres mots, les structures spatiales ont souvent le rôle d'un élément organisateur, le rôle modélisant autour duquel s'assemblent les éléments non-spatiaux au sein d'un texte littéraire.

La notion centrale de cette approche est celle de la frontière divisant l'espace d'un récit en deux sous-espaces. ${ }^{1}$ Ce qui est important d'ailleurs est que « la frontière qui divise un espace en deux parties doit être impénétrable, et la structure interne de chaque sous-espace, différente » (Lotman 1973 : 321). En plus, cette division spatiale est souvent nette - comme c'est le cas du roman Le rivage des Syrtes, où la frontière dont le passage est interdit est représentée par la limite maritime entre deux pays en guerre. Elle est marquée nettement sur les cartes maritimes par une ligne rouge. En général, ces frontières qui conviennent aux héros à les franchir et transgresser font partie d'une unité supérieure que Lotman appelle le continuum spatial du texte (1973: 321). Celui-ci ne se réduit pas à la présentation des lieux et des paysages seulement comme un arrière-plan décoratif où se déroulent les actions des personnages. En fait, la spatialité d'un texte reproduisant le monde matériel s'ordonne en un topos littéraire caractérisé par sa propre structure créée elle-même par un système de relations et d'organisations spatiales. C'est-à-dire que la structure même du topos fonctionne comme le modèle pour exprimer les relations contenus dans le texte qui n'ont pas, autrement, une nature spatiale. Dans Le rivage des Syrtes il s'agit par exemple du topos du lieu interdit, le Farghestan ennemi, dont la structuration participe à la construction du thème de l'initiation.

Le Farghestan avait dressé devant moi [Aldo] des brisants de rêve, l’au-delà fabuleux d'une mer interdite [...] (Gracq $1951: 217)$.

1 Le cas où l'espace du texte est divisé par une frontière en deux parties est le plus fondamental. Il arrive assez souvent que la structure de la division soit plus complexe. Pourtant, nous croyons que ce modèle simple servira suffisamment le but qui est le nôtre - une démonstration du principe de fonctionnement de la relation de la formation de l'espace littéraire et de ses conséquences pour la portée sémantique du roman analysé. 
C’est grâce au déplacement du personnage principal, Aldo, dans cette structure caractérisé par des oppositions binaires de l'ici et de l'au-delà (la Seigneurie d'Orsenna et le Farghestan, le connu et l'inconnu/l'interdit) que progresse sa recherche identitaire. De cette façon il peut finalement aboutir à un acte important dans le processus initiatique, une réconciliation des contraires qui sont l'ignorance et la connaissance, la vie et la mort.

$\mathrm{Au}$ concept de l'espace artistique dans la narration est étroitement lié le concept thématique fondamental, lévénement. Or, une question se pose : que représente l'évènement en tant qu'unité de la structure thématique ? Selon Lotman, « l'événement dans le texte est le déplacement du personnage à travers la frontière du champ sémantique» (1973 : 326). Et ce mouvement est constitué par le fait de traverser une frontière spatiale chargé d'interdit.

Ce qui résulte de cette brève présentation de l'optique lotmanienne pour l'œuvre romanesque de Julien Gracq est que la fragmentation de l'espace en tant que mécanisme de narration est importante au niveau de la diégèse ${ }^{2}$. Ainsi, la modélisation des lieux parcourus par les héros de Gracq est étroitement liée au niveau symbolique de ces lieux et l'acte de traverser une frontière dans le monde matériel a, maintes fois, une signification sémantique d'importance et représente un événement au niveau thématique et narratif. L'espace et les frontières qui le découpent et le délimitent apparaissent tantôt comme réels, tantôt comme symboliques. Effectivement, chez Gracq, la frontière classificatrice divisant les champs sémantiques opposés a souvent le caractère d'une frontière spatiale que le héros doit traverser afin d'accomplir sa quête initiatique. L'auteur lui-même a jadis exprimé sa prédilection pour les frontières lors de son entretien avec Jean Carrière (1986 : 157) :

Ces confins, lisières, frontières, effectivement sont des lieux qui m'attirent en imagination : ce sont des lieux sous tension, et peut-être cette tension est-elle matérialisée, localisée - l'équivalent de ce qu'est la tension latente entre ses personnages pour un romancier psychologue : un stimulant imaginatif. [...] Il arrive le plus souvent que ces personnages dans les romans soient euxmêmes mis, par rapport à la société dans une situation de lisière par une guerre, par des vacances, par une mise en disponibilité quelconque.

Il est vrai que le héros gracquien est, en général, un être éprouvant la solitude, le sentiment du vide et du manque et ces sentiments inspirent son déplacement dans l'espace, ses errances et sa recherche d'un au-delà. Il s'agit d'un marcheur solitaire défini par Mircea Eliade comme :

[...] celui qui par sa marche, son ascèse [...] exprime symboliquement que l'extérieur et l'intérieur n'en font qu'un et que la conquête de l'espace intérieur est indissociable de celle de l'espace géographique (Thomas $1998: 115$ ).

Aldo, lui aussi, entretient une relation de curiosité et de désir avec son environnement. Entre lui et le paysage, le contact physique, la marche, et le contact imaginaire sous forme de rêveries, projections, désirs et prémonitions constituent un processus de connaissance. Le pays au-delà

2 Dans les mécanismes de narration, la diégèse est le fait de raconter les choses, et s'oppose au principe de mimesis qui consiste à montrer les choses. 
de la limite maritime se présente comme une porte ouverte sur l'imaginaire, mais qui se propose aussi comme un espace à découvrir. Il l'invite à dépasser les frontières, même s'il devrait s'agir d’un acte condamnable par la société.

Orsenna et le monde habitable finissaient à cette frontière d'alarme [...]. J'étais [Aldo] prêt à douer de prodiges concrets ce passage périlleux, à m’imaginer une crevasse dans la mer, un signe avertisseur $[\ldots]$. Très au-delà, prodigieux déloignement derrière cet interdit magique, sétendaient les espaces inconnus du Farghestan, serrés comme une terre sainte à lombre du volcan Tängri [...] (Gracq $1951: 34)$.

Dès lors, l'acte de transgression de l'interdit, du seuil divisant le passé et l'avenir se prépare. Il exigera une rupture avec un état actuel, au niveau collectif du destin des deux pays en guerre, mais aussi au niveau personnel du héros, et l'accession à un nouvel état des choses et à la révélation. L'aventure d'Aldo commence ainsi par la reconnaissance d'un seuil qui a, en plus, le caractère d'une démarcation entre la sphère de l'interdit et du caché. L'espace au-delà de ce seuil exerce sur lui un pouvoir magnétisant. Déjà la prise de conscience de cette frontière ouvre la voie au déclenchement de sa transformation intérieure. Or, la quête du sens de la vie est étroitement liée à l'interdit, représenté par une frontière spatiale qui doit être dépassée et qui est, en même temps, un seuil initiatique. Le désir éprouvé devant cette frontière est le moteur pour passer de l'état de contemplation à l'action. En fait, Aldo qui avait été assigné au poste d'Observateur dans la province éloignée et côtière des Syrtes, se trouve devant un seuil interdit à plusieurs reprises. D’abord, il succombe à la curiosité et au désir d'explorer la chambre des cartes à l'Amirauté. Cette salle représente un seuil symbolique car, en le dépassant, le héros entre dans un champ sémantique dont la structure est différente de ce qu'il avait connu jusque-là ; elle offre le contact avec le mystère et l'interdit. Dans la chambre, Aldo découvre les cartes maritimes marquées par une ligne rouge.

Devant moi sétendaient en nappe blanche les terres stériles des Syrtes, piquées des mouchetures de leurs rares fermes isolées, bordées de la délicate guipure des flèches des lagunes. Parallèlement à la côte courait à quelque distance, sur la mer, une ligne pointillée noire : la limite de la zone des patrouilles. Plus loin encore, une ligne continue d'un rouge vif : cétait celle, qu'on avait depuis longtemps acceptée d'un accord tacite pour ligne frontière, et que les instructions nautiques interdisaient de franchir en quelque cas ce fût (Gracq $1951: 33$ ).

Cette division spatiale sert comme une démarcation des deux pays ennemis en guerre assoupie ; la Seigneurie d'Orsenna, en état de déclin, de stagnation, de délabrement et d'ignorance et le pays du Fargherstan symbolisant un lieu possible de la connaissance révélatrice et de l'initiation. Cette découverte va avoir une importance capitale dans le parcours initiatique d'Aldo, car le mouvement entre les deux espaces appartenant aux champs sémantique de caractère opposé, et le final acte libérateur de la transgression de la frontière maritime entraîneront la métamorphose existentielle de l'Observateur.

Pour une démonstration plus complexe du sémantisme spatial chez Gracq, comparons le roman étudié jusqu' ici avec un autre roman de l'auteur, Un balcon en forêt (2011). Ici, la frontière 
divisant l'espace en deux sous-espaces sémantiquement différents, est présente aussi. Il s’agit de la démarcation matérialisée par la lisière de la forêt. La division signale la sphère du réel marquée par l'approche irréversible de la guerre et l'espace de la rêverie éveillée et du mythique archétypal personnifiant l'inconscient du personnage de Grange. L’aspirant Grange, héros principal, qui fait son service militaire près de la frontière belge, passe ses jours patrouillant dans les forêts ardennaises. Ses promenades entre le monde de la conscience et le monde de l'inconscient, métaphoriquement parlant, deviennent le moyen d'atteindre une unité existentielle. En descendant dans les couches profondes de sa psyché pendant les virées forestières, il effectue, ultérieurement, une remontée vers la connaissance. Cette révélation finale s'accomplit pendant le temps qu’il repose, mortellement blessé, dans la maison de sa maitresse Mona, au sein de la forêt ${ }^{3}$.

Les deux textes ont en commun non seulement la présence des frontières sémantiques matérialisées dans le monde des objets, mais aussi des affinités au niveau mental des héros principaux. Les deux personnages partagent un pressentiment que quelque chose d'une importance capitale pour leurs vies va arriver. Tandis qu'Aldo parle d'une promesse,

[...] Quelque chose m’était promis, quelque chose m’était dévoilé ; j’entrais sans éclaircissement dans une intimité presque angoissante, j’attendais le matin offert déjà de tous mes yeux aveugles, comme on s'avance les yeux bandés vers le lieu de la révélation (Gracq 1951 : 19).

la prémonition de Grange est plus vague :

J'avais peur et envie, se dit-il. J'attendais que quelque chose arrive. J'avais fait de la place pour quelque chose... Il savait bien que quelque chose était arrivé, mais il lui semblait que ce ne fût pas réellement (Gracq $2011: 250$ ).

Ces sentiments d'Aldo et de Grange trouveront leur accomplissement dans la prise de conscience que la reconnaissance d'une frontière, d'abord, et son dépassement, ensuite, conditionnent leur évolution ultérieure. Et cette révélation fait en sorte que les deux personnages sont attirés irrésistiblement par ce seuil et le désir de la transgression. Pour Aldo, il s'agit d'une tentation personnifiée par le fantôme Farghestan qui motive sa croisière maritime :

Le Farghestan avait dressé devant moi des brisants de rêve, l’au-delà fabuleux d'une mer interdite [...] La dernière tentation, la tentation sans remède, prenait corps dans ce fantôme saisissable, dans cette proie endormie sous les doigts déjà ouverts (Gracq 1951 : 217-218).

Grange, quant à lui, est attiré par la lisière de la forêt :

[...] cette lisière sourdement alertée où les forêts patrouillées de la guerre venaient border on ne savait quel silence respirant et heureux que tendait l'oreille attirait Grange et l'intriguait (Gracq $2011: 96)$.

3 L'importance symbolique que peut avoir l'espace forestière pour le processus de la transformation ontologique de l'être confirme aussi Mircea Eliade en disant que « la forêt est un symbole de l'au-delà, nous le retrouvons dans nombreux rites initiatiques " $(1957: 237)$. 
Tandis que la transgression de la limite maritime, acte illicite d'Aldo, peut être considérée comme un acte libérateur et héroïque au sens du destin collectif de la Seigneurie d'Orsenna, la transgression de Grange est étroitement liée à la régression personnelle. Aldo accomplit sa transgression d'un seul coup et ainsi il met fin à l'inertie mortelle de son pays, tandis que Grange effectue la sienne successivement, chaque fois qu'il dépasse la frontière de la forêt et plonge toujours plus profondément dans son inconscient. Le principe du mouvement entre deux espaces sémantiquement et structurellement différents est similaire, mais la motivation des personnages diffère ; Aldo est activé par le désir du changement, Grange par la volonté du lâchez tout ${ }^{4}$ et la régression, d'un retour à l'état de l'enfance, à l'unité originaire. Le résultat est pourtant le même - la rupture avec l'état antérieur de l'ignorance et l'aspiration à une existence nouvelle. Pour Grange, il s'agit de larguer ses attaches :

[...] il lui semblait qu'il larguait ses attaches; il entrait dans un monde racheté, lavé de l'homme, collé à son ciel d’étoiles de ce même soulèvement pâmé qu’on les océans vides. «Il n’y a que moi au monde » se disait-il avec une allégresse qui l'emportait » (Gracq 2011 : 97).

Pour Aldo, c'est plutôt le cas du désir « de passer vers l’au-delà interdit » (Gracq 1951 : 217), de commettre un acte illicite. Ainsi, lors de la nuit du passage au-delà de la limite maritime il éprouve des sensations extraordinaires :

Il me semblait que nous venions de pousser une de ces portes qu'on franchit en rêve. Le sentiment suffocant d'une allégresse perdue depuis l'enfance s'emparait de moi [...] il me semblait que maintenant tout entier, j'étais remis - une liberté, une simplicité miraculeuse lavaient le monde. [...] Il me semblait que soudain le pouvoir m’êt donné de passer outre (Gracq 1951 : 223).

Dans les deux cas il s'agit du dépassement des frontières spatiales, correspondant aux frontières sémantiques au sein d'un continuum spatial du texte. Or, le mouvement dans l'espace modelé, afin de prêter le sens aux concepts qui nont pas en soi une nature spatiale, et la transgression, en tant que l'événement selon la définition de Lotman, se montrent comme des actes catalyseurs dans la structuration thématique des romans, c'est-à-dire dans la quête de la recherche d'une unité primordiale perdue et d'un état susceptible de concilier les contradictions des mondes opposées (réalité et rêve, guerre et paix, ignorance et révélation). Ainsi, devient évidente que lévénement, transgression de la frontière sémantique, est une condition de la progression de l'action romanesque vers le dénouement final consistant en l'accomplissement du parcours initiatique. Autrement dit, la conquête de l'espace intérieur des héros est indissociable de celle de la conquête de l'espace géographique (et de la transgression d'un seuil interdit). Or, les frontières spatiales correspondent aux frontières sémantiques textuelles et les deux assument le rôle de signifiant au niveau du développement du sens.

4 Le désir de "lâchez tout ", ce sentiment de la liberté intérieure, est étroitement lié avec une possibilité de la transgression des frontières imposées par la vie et les devoirs du soldat. Il est lié avec « ce besoin de faire sauter une à une les amarres ». Il s'agit d’un sentiment " de délestage et de la légèreté profonde qui faisait bondir le cœur et qui était celui du lâchez tout » (Gracq 2011 : 212). 


\section{Références bibliographiques}

Thomas, J. (1998). Introduction aux méthodologies de l'imaginaire. Paris : Ellipses.

Carrière, J. (1986). Julien Gracq. Qui êtes-vous? Lyon : La Manufacture.

Eliade, M. (1957). Mythes, rêves et mystères. Paris : Éditions Gallimard.

Gracq, J. (1951). Le Rivage des Syrtes. Paris : Librairie José Corti.

. (2011). Un Balcon en forêt. Paris : Librairie José Corti.

Lotman, I. (1973). La structure du texte artistique. Paris : Éditions Gallimard. 
